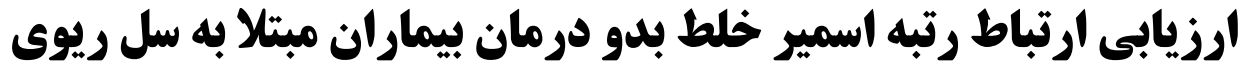

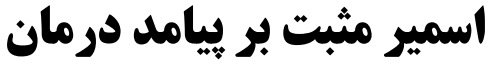

\author{
مهران يارى'، ليلا ملائى ثور '، محمدرضا جعفرى'، على اصغر ولى يور'، سلمان خزايى ‘*، قدرت الله روشنايىه \\ ا I. كميته تحقيقات دانشجويى، دانشكده علوم يزشكى آبادان، آبادان، ايران

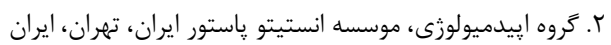

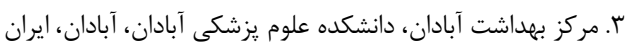

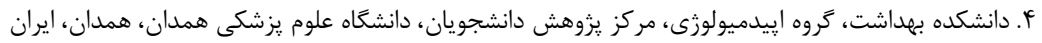

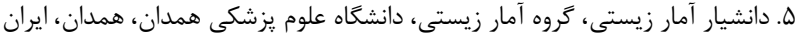

\section{olve}

مقدمه: درمان ناموفق سل يك مشكل جدى براى بهداشت عمومى است. نتايج متناقضى در خصوص

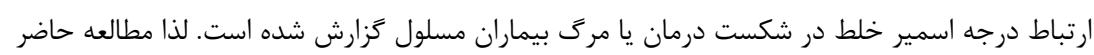
با هدف ارتباط رتبه اسمير خلط بيماران در بدو درمان بر پيامد درمانى آنها انجام شد.

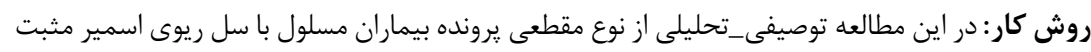

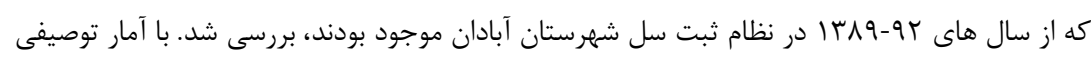

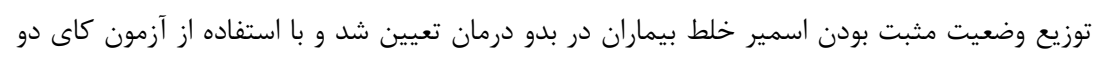

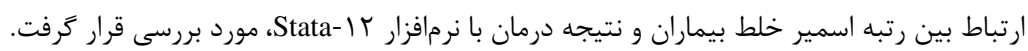

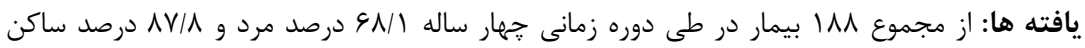

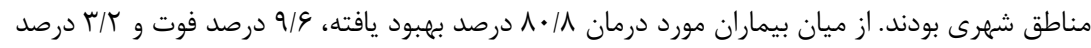
دجار شكست درمان شده بودند، و r/V درصد نيز انتقال يافته به ساير دانشعاه ها و T/V درصد نيز غيبت از درمان داشتند. همجنين نتايج نشان داد كه بين سطح باسيل در ابتداى درمان با ٍِيامد درمانى بيماران

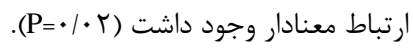

نتيجه كَيرى: نتايج نشان داد كه رتبه مثبت بودن اسمير اوليه خلط ارتباط معنى دارى با پيامد درمان دارد، به نحوى كه در بيماران با رتبه بالاى اسمير خلط در بدو درمان ميزان بهبودى كمتر است. لذا توصيه مى كردد ارائه درمان استاندارد تحت شرايط مناسب و صحيح مديريتى شامل نظارت مستقيم روزانه و حمايت بيماران تا بهبودى كامل صورت كيرد.

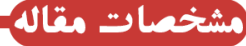

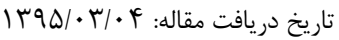

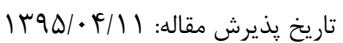

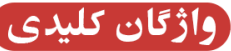

$$
\text { سل }
$$$$
\text { خلط }
$$

بيامد درمان

\section{تويسنلده مسنول}

سلمان خزايى، دانشكده بهداشت، كروه

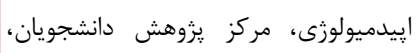
دانشَاه علوم يزشكى همدان، همدان، ايران

تلفن:

ايميل: S_khazaeii@Yahoo.com

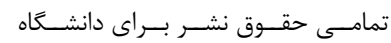

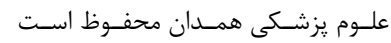

مقلدمه:

بيمارى سل مى شوند [[]. سل به عنوان دومين علت مرگى و

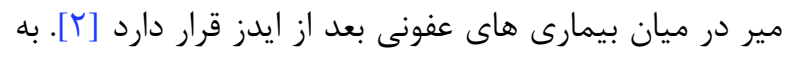

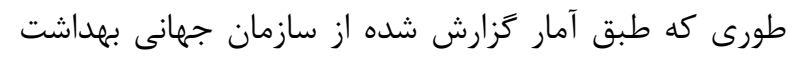

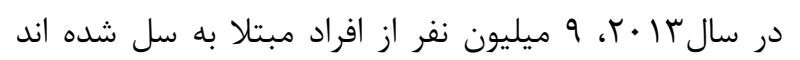

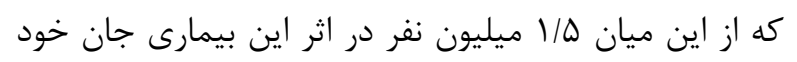

سل يكى از بيمارى هاى عفونى بسيار شايع در جهان است

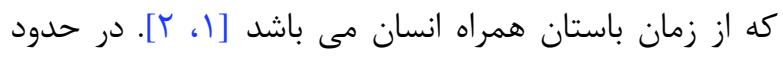

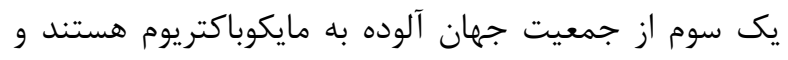

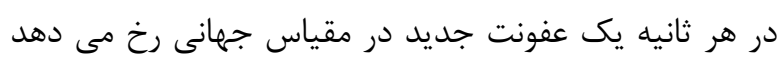

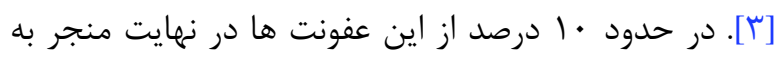


سن بيش از له سال، جنسيت مرد، عوارض جانبى دارو، سن

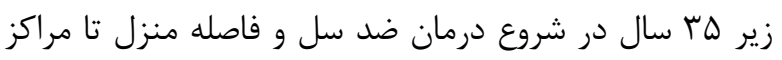

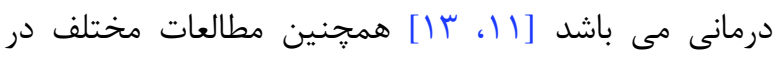

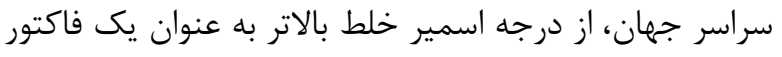

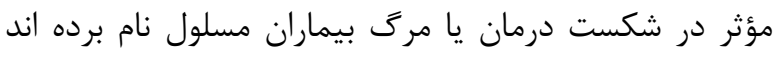

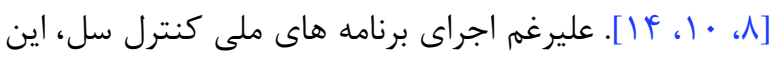

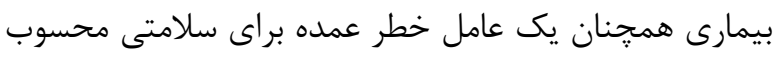

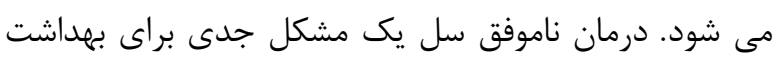

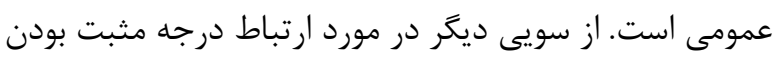

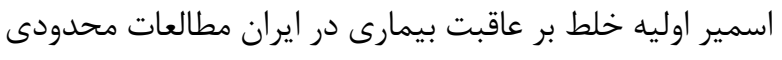

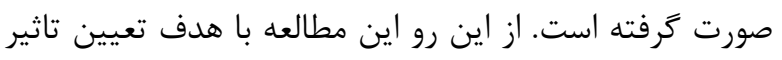

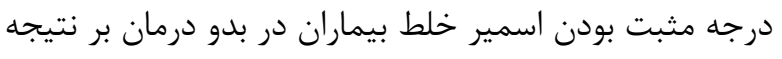

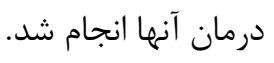

\section{مواد و روش ها}

اين مطالعه توصيفى_تحليلى در شهرستان آبادان در طى سال

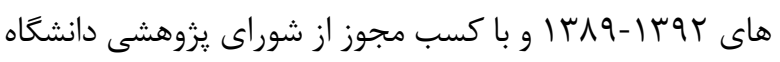

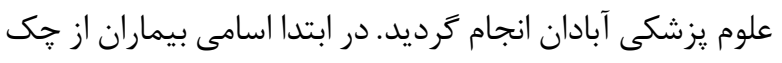

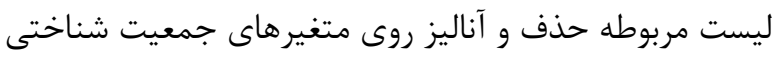

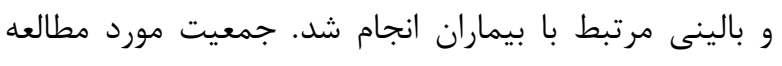

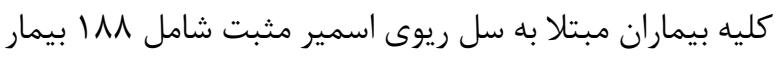

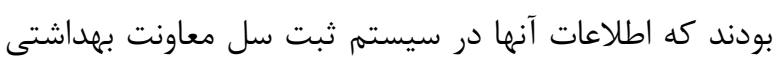

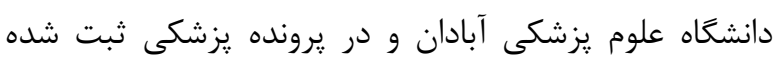

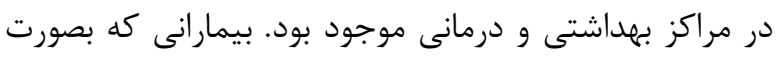

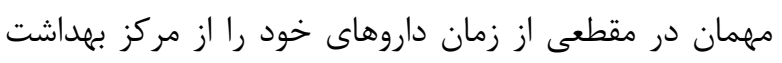

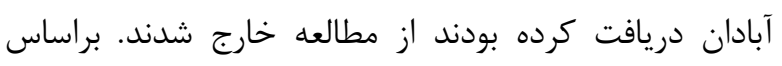

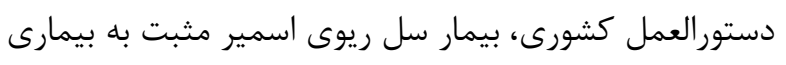

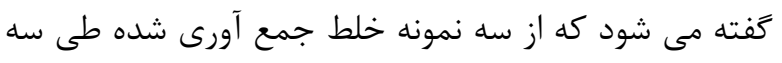

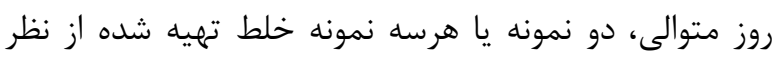

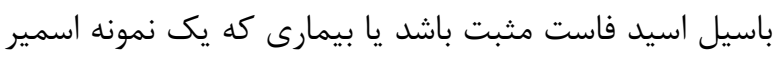

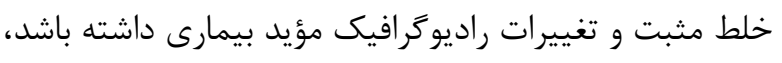

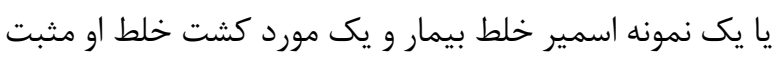

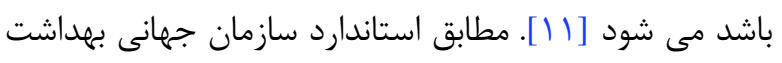

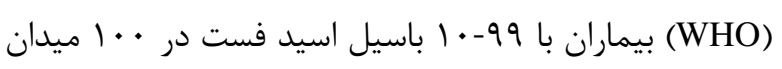

را از دست داده اند [r]]. همرنين بار بيمارى بيشتر متوجه كشورهاى درحال توسعه و با درآمد كم و متوسط است و بيش از هو درصد از موارد مرك و مير ناشى از سل در اين كشورها

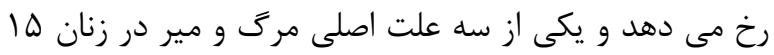

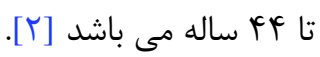
سازمان جهانى بهداشت با صعودى شدن روند بار جهانى سل،

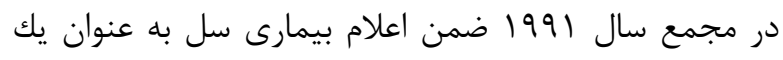
اورزانس جهانى، كاهش هر جه سريعتر ميزان شيوع، مرى و و

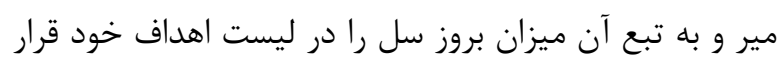

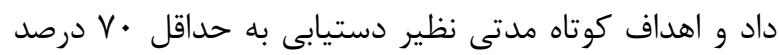

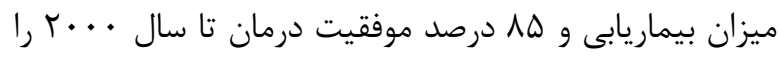
تعيين و به كشورها ابلاغ نمود، سيس با معرفى راهبرد DOTS Directly Observed Treatment Short-Course بيمارى و دستيابى به اين اهداف را به طور نسبى فراهم آورد.

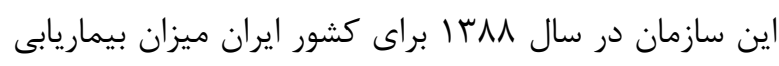

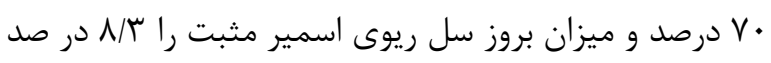
هزار نفر در نظر گرفته بود [ه، 9].

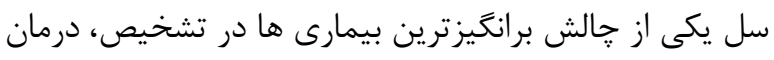

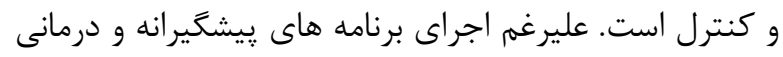

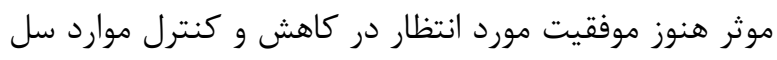

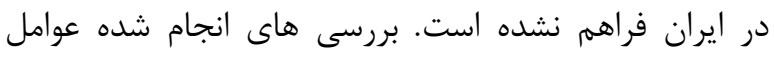

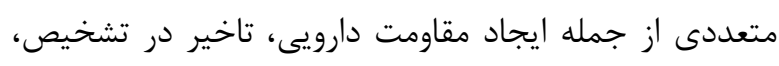

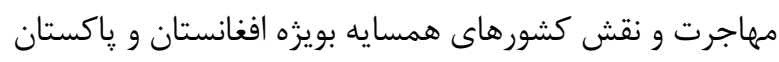
در بروز موارد جديد، فقر، قوميت، جنسيت، تغذيه نامناسب،

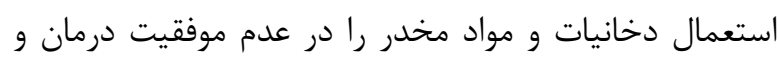
كنترل بيمارى موثر دانسته اند [V، • [1].

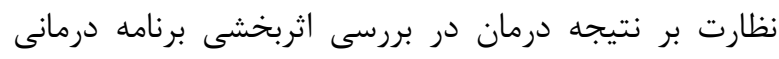

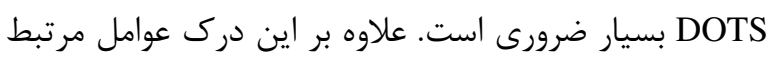

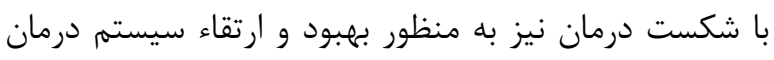
DOTS

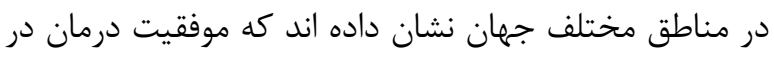

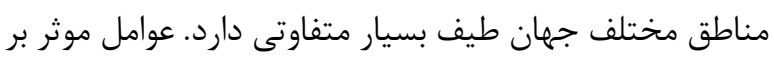

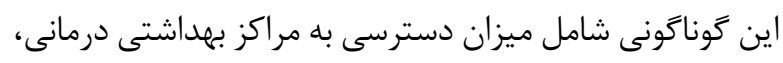

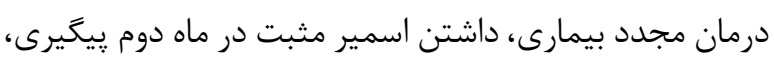


ميكروسكويى به عنوان +1، · (-1 باسيل اسيد فست در هر در محدوده سنى 9 تا 19 سال قرار داشتند و فقط 9•/ آنها

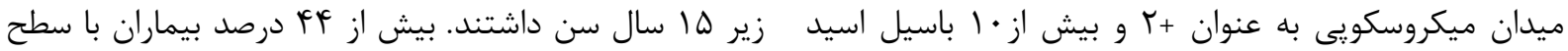

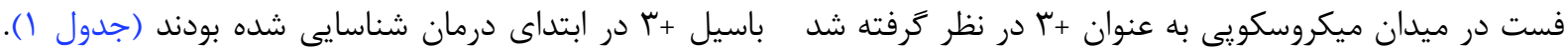

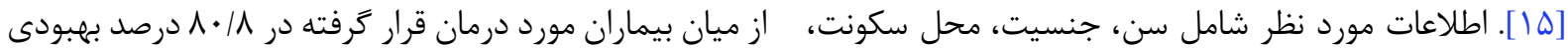

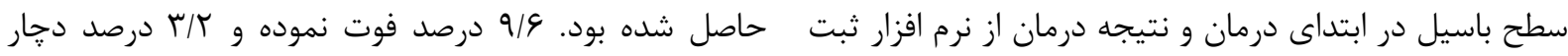

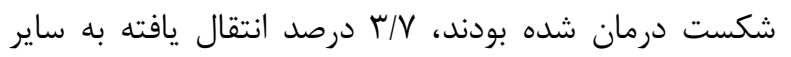

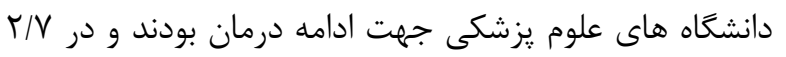

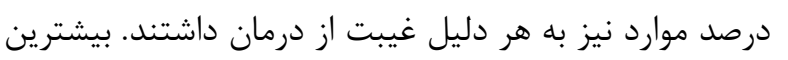

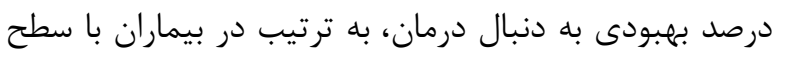

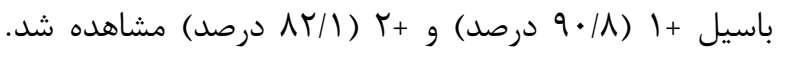
كمترين و بيشترين درصد مرك به دنبال دريافت درمان به بيماران سل موجود در معاونت بهداشتى استخراج ترديد.

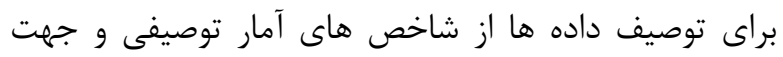

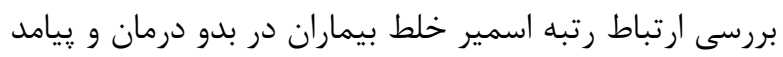

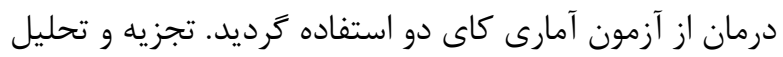

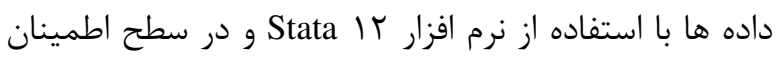

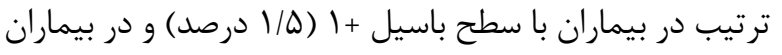

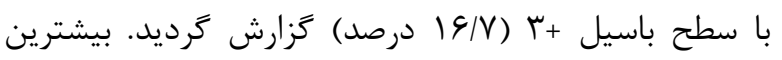

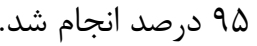

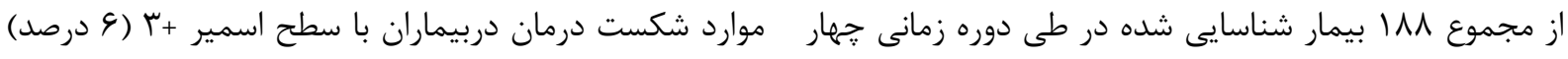

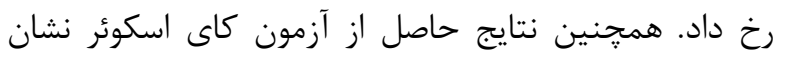

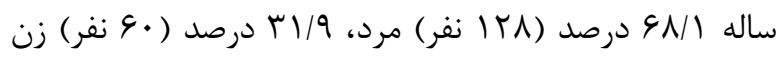

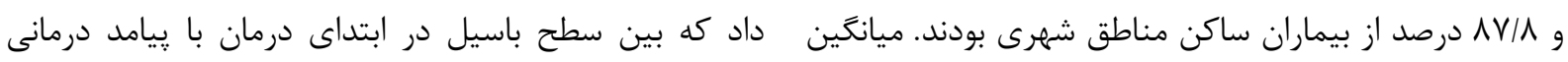

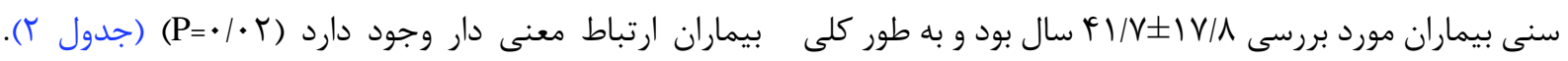

\begin{tabular}{|c|c|c|}
\hline فراوانى (درصد) & & متغير \\
\hline $\mid r \Lambda(9 N / I)$ & مرد & \multirow[b]{2}{*}{ جنس } \\
\hline$\varphi \cdot(r) / 9)$ & 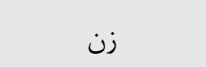 & \\
\hline $190(\Lambda V / \Lambda)$ & شهر ا & \multirow[b]{2}{*}{ منطقه سكونت } \\
\hline$r(I T / Y)$ & روستا & \\
\hline$\varphi \Delta(r / / \varphi)$ & $1+$ & \multirow{3}{*}{ سطح باسيل در ابتداى درمان } \\
\hline rq(r./V) & $r_{+}$ & \\
\hline$\lambda f(F / V)$ & $r_{+}$ & \\
\hline$r(1 / 1)$ & كمتر از هاسال & \multirow{4}{*}{ 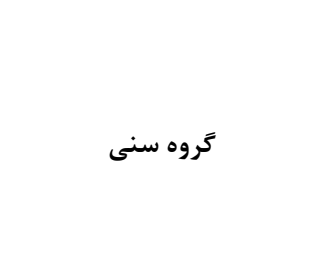 } \\
\hline$\Delta T(T V / Q)$ & $1 \Delta-r$. & \\
\hline$V F(r q / q)$ & rI-ब. & \\
\hline $4 \cdot(\Gamma) / 9)$ & بيشتر از •ه سال & \\
\hline
\end{tabular}




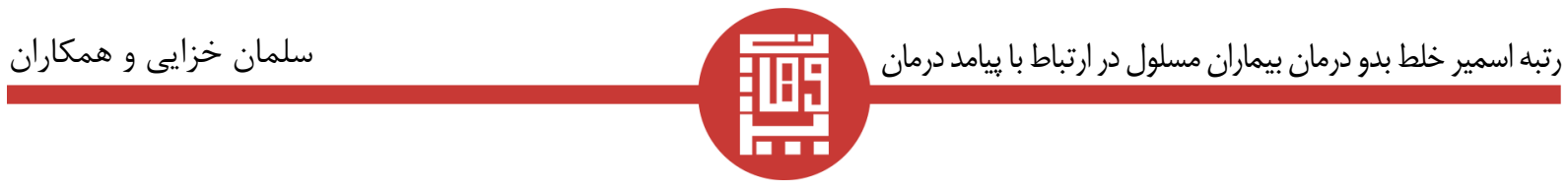

جدول r. ارتباط بين سطح باسيل در ابتداى درمان با نتيجه درمانى

\begin{tabular}{|c|c|c|c|c|c|c|}
\hline \multirow{3}{*}{ سطح معنادارى*** } & \multirow{3}{*}{ مجموع } & \multicolumn{4}{|c|}{ نتيجه درمان } & \\
\hline & & \multicolumn{3}{|c|}{ عدم بهبودى } & \multirow[b]{2}{*}{ بهبودى } & \\
\hline & & ساير* & شكست & مرى & & \\
\hline & $G \Delta(r Y / \Delta V)$ & $\Delta(V / V)$ & $\cdot(\cdot)$ & $I(1 / Q F)$ & $\Delta q(q \cdot / V \vee)$ & $1+$ \\
\hline \multirow[t]{2}{*}{$\mathrm{P}=\cdot / \cdot r$} & $r q(r \cdot / V F)$ & $r(v / 9 q)$ & $1(\Gamma / \Delta \varphi)$ & $r(V / 99)$ & $\operatorname{Tr}(\lambda T / \cdot \Delta)$ & $r_{+}$ \\
\hline & $\Lambda F(F F / G \Lambda)$ & $\mathcal{F}(\mathcal{F} / V Y)$ & $\Delta(\Delta / 9 \Delta)$ & $\mid f(|\&| q V)$ & GI (VT/GY) & $r+$ \\
\hline
\end{tabular}

خطر انتقال گسترده تر يا فعال سل و تظاهرات بالينى بيشتر در

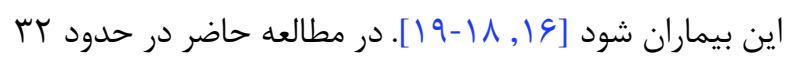

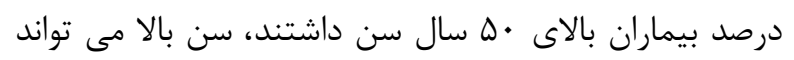

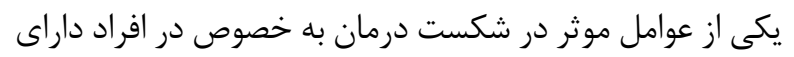
باسيل بيشتر باشد. مطالعه Fahrettin Talay در كشور تركيه نشان داد كه سن بالاى \&\$ سال يك عامل موثر در شكست درمان بيماران مسلول مى باشد [·r]]. همه مطالعات فوق در توافق با مطالعه ما، درجه مثبت بودن

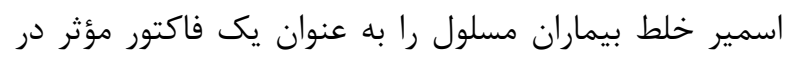

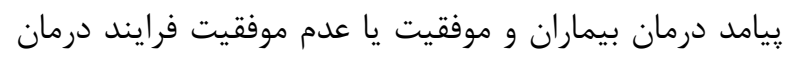

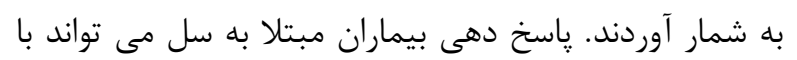

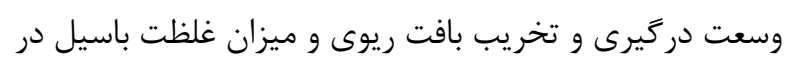

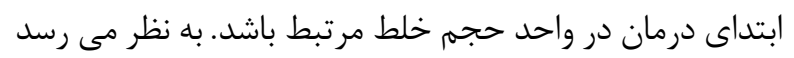

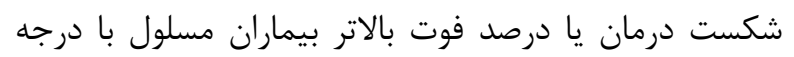
اسمير خلط بالاتر در ابتداى درمان، با تخريب شديدتر بافت و بان حفره ريه در ارتباط باشد. اين مطالعه با يك محدوديت مواجه بود و آن اين است كه داده هاى مورد نياز اين يزوهش از اطلاعات ثبت شده در سيستم

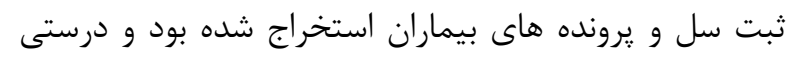

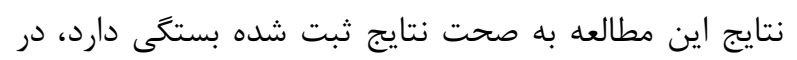

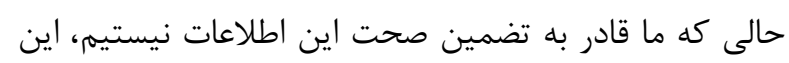
مساله خود ممكن است منجر به سوگيرى اطلاعات كردد. از جمله نقاط قوت اين مطالعه مى توان بيان كرد كه جزء محدود
بيشترين تعداد بيماران مورد بررسى در اين مطالعه در ابتداى

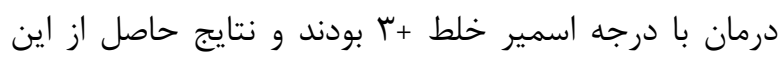

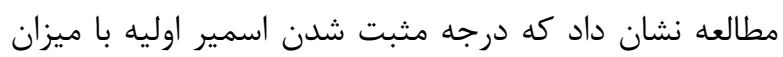
بهبودى رابطه عكس دارد به طورى كه تنها در حدود • V درصد از بيماران با سطح باسيل +r يس از دريافت درمان بهبود يافته بودند، درحالى كه بيش از • 9 درصد از بيماران با سطح باسيل +1، پس از درمان بهبودى كامل يافته بودند. مطالعه اى كه توسط فرازى و همكاران در سال دي إد در استان مركزى انجام شد نشان داد كه بيشترين موارد مثبت شدن اسمير خلط بعد

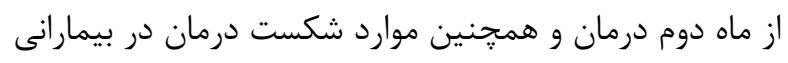

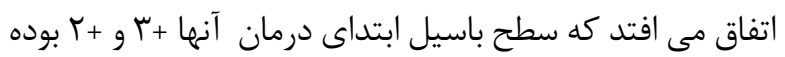

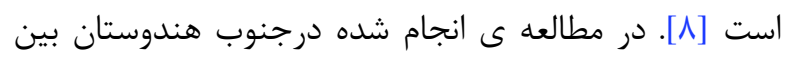

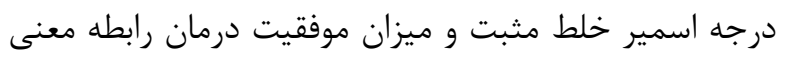

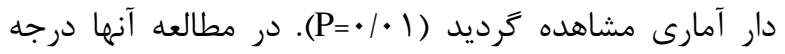
اسمير مثبت خلط با پيامد درمان رابطه خطى داشت و نسبت بيماران با پيامد درمان نامطلوب، در بين بيماران داراى اسمير

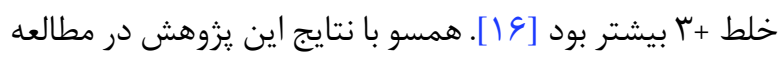

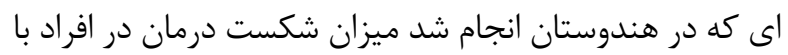

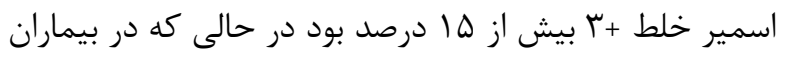

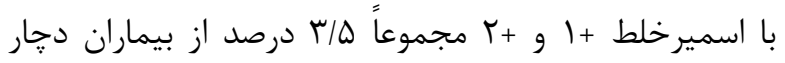

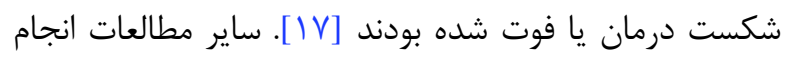
شده نشان دادند كه بالا بودن درجه اسمير خلط مثبت بيماران علاوه بر تاثير معكوس بر يِيامد درمان مى تواند منجر به افزايش 
مطالعاتى الست كه در سطح كثور در اين خصوص انحام شده مطلوب فاصله دارد. همحنين اين مطالعه نشان داد كه درجه است و نتايج مطالعه مى تواند در مديريت بالينى بيماران مبتلا مثبت بودن اسمير اوليه خلط ارتباط معنى دارى با درجه

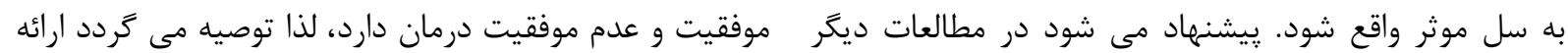
و در نقاط مختلف كشور به تاثير و نقش سن و ساير عوامل درمان استاندارد تحت شرايط مناسب و صحيح مديريتى شامل بالينى و جمعيت شناختى بيماران مسلول بر زيامد هاى درمان نظارت مستقيم روزانه (DOTS) و حمايت بيماران تا بهبودى

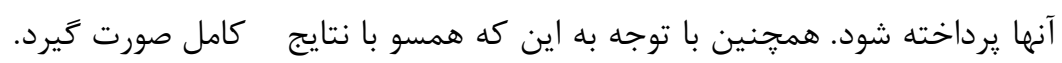

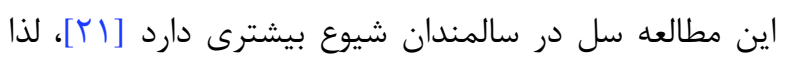

\section{تقلدير و تشكر}

نويسندكان اين مقاله بر خود لازم مى دانند از يزشكان و

كارشناسان درگير در برنامه بيشخيرى و كنترل سل شهرستان

$$
\text { آبادان تقدير و تشكر به عمل بياورند. }
$$

\section{تضاد منافع}

اين مطالعه براى نويسندگان هيج گونه تضاد منافعى نداشته است.

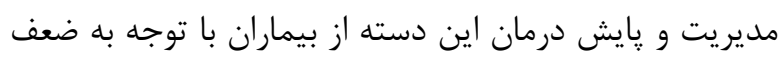
قواى جسمانى و سيستم ايمنى بدن و همجنين وابستكى به اطرافيان براى پيخيرى درمان مى بايست با حساسيت بيشترى دنبال شود.

\section{نتيجه كيرى}

ميزان بهبودى به دنبال درمان در بيماران مسلول در اين

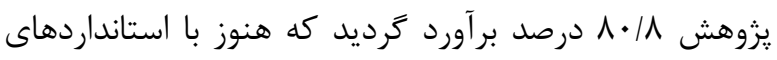

\section{References}

1. Lawn S, Zumla A. Tuberculosis. The Lancet. 2011;378(9785):57-72.

2. World Health Organization. Tuberculosis. WHO; 2015 [updated March, 2015; cited May, 2015]; Available from: http://www.who.int/mediacentre/ factsheets/fs 104/en/.

3. World Health Organization. Strengthening health sector response to HIV/AIDS and sexually transmitted infections in the Eastern Mediterranean Region 20062010. WHO; 2006 [updated June, 2006; cited May, 2015]; Available from: http://applications.emro.who. int/aiecf/who_em_std_089_en.pdf.

4. Centers for Disease Control and Prevention. Tuberculosis (TB). 2012 [updated May, 2015; cited September, 2015]; Available from:http://www.cdc.gov/ tb/publications/factsheets/general/LTBIandActiveTB.

html.

5. World Health Organization. Global Tuberculosis Control 2010, WHO; 2010 [cited September, 2015]; Available from:http://reliefweb.int/.../ F530290AD0279399C12577D8003E9D65-Full_ Report.pdf.

6. Nasehi M. Guideline for control Tuberculosis. $1^{\text {st }} \mathrm{ed}$. Tehran:Andishmand;2009. 5-21. (Persian)

7. Gopi PG, Vasantha M, Muniyandi M, Chandrasekaran V, Balasubramanian R, Narayanan PR. Risk factors for non-adherence to directly observed treatment (DOT) in a rural Tuberculosis unit, South India. Indian Journal of Tuberculosis. 2007;54(2):66-70.

8. Epstein MD, Schluger NW, Davidow AL, Bonk S, Rom WN, Hanna B. Time to detection of Mycobacterium Tuberculosis in sputum culture correlates with 


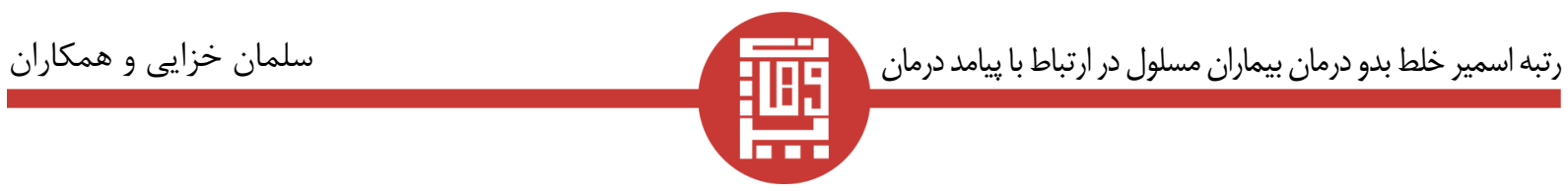

outcome in patients receiving treatment for pulmonary Tuberculosis. Chest Journal. 1998;113(2):379-386.

9. Telzak EE, Fazal BA, Pollard CL, Turett GS, Justman JE, Blum S. Factors influencing time to sputum conversion among patients with smear-positive pulmonary Tuberculosis. Clinical Infectious Diseases. 1997;25(3):666-670.

10. Vasudevan K, Jayakumar N, Gnanasekaran D. Smear conversion, treatment outcomes and the time of default in registered Tuberculosis patients on RNTCP DOTS in puducherry, Southern India. Journal of Clinical and Diagnostic Research. 2014;8(10):5-8.

11. Ministry of Health and Medical Education. National guide for the care and treatment of HIV/AIDS. Tehran:MOHME;2012. (Persian)

12. Gopi PG, Chandrasekaran V, Subramani R, Santha

T, Thomas A, Selvakumar N, et al. Association of conversion \& cure with initial smear grading among new smear positive pulmonary Tuberculosis patients treated with category i regimen. The Indian Journal of Medical Research. 2006;123(6):807-814.

13. Kanade S, Nataraj G, Anita R, Mehta P. Correlation between smear positivity grade at two months with culture positivity and final outcome in patients receiving antituberculosis treatment. Bombay Hospital Journal. 2010;52(2):183-188.

14. Amante TD, Ahemed TA. Risk factors for unsuccessful Tuberculosis treatment outcome (failure, default and death) in public health institutions, Eastern Ethiopia. The Pan African Medical Journal. 2015;20:247. Epub 2015/07/15.
15. Marjani M, Baghaei P, Tabarsi P, Shamaei M, Mansouri D, Masjedi M, et al. Drug Resistance pattern and outcome of treatment in recurrent episodes of Tuberculosis. Eastern Mediterranean Health Journal. 2012;18(9):957-961. 16. Jha UM, Satyanarayana S, Dewan PK, Chadha S, Wares F, et al. Risk factors for treatment default among re-treatment Tuberculosis patients in India, 2006. PLoS ONE. 2010;5(1):1-7.

17. Zadeh JH, Nasehi M, Rezaianzadeh A, Tabatabaee H, Rajaeifard A, Ghaderi E. Pattern of reported Tuberculosis cases in iran 2009-2010. Iranian Journal of Public Health. 2013;42(1):72-78. (Persian)

18. Pardeshi GS. Time of default in Tuberculosis Patients on directly observed treatment.Journal of Global Infectious Diseases. 2010;2(3):226-230.

19. Khazaei S, Zahiri A, Hasanbeigi A, MohammadianMafshejani A, Salehiniya H, Saatchi M et al . Effect of intensity of sputum smear positive in pulmonary Tuberculosis patients at the beginning of treatment on treatment outcome. Journal of Ilam University of Medical Sciences. 2016;24(1):1-7. (Persian)

20. Talay F, Kumbetli S, Altin S. Factors associated with treatment success for Tuberculosis patients: a single center's experience in Turkey. Japanese Journal of Infectious Diseases. 2008;61(1):25-30.

21. Najafi Vosogh R, Roshanaei G, Khazaei S, Safari M, Zahiri A, Bothaei J. Study of Tuberculosis epidemiology and its affected factors in Hamadan province, during the years 2007-2013. Pajouhan Scientific Journal. 2015;14(1):64-71. (Persian) 


\title{
Assessing relation between sputum smear grading in positive pulmo- nary Tuberculosis patients with treatment outcome
}

\author{
Mehran Yari ${ }^{1}$, Leila Molaeipour ${ }^{2}$, Mohammad Reza Jafari ${ }^{3}$, Ali Asghar Valipour ${ }^{1}$ \\ , Salman Khazaei ${ }^{4 *}$, Ghodratollah Roushanaei ${ }^{5}$ \\ 1. Student Research Committee, Abadan Faculty of Medical Sciences, Abadan, Iran \\ 2. Department of Epidemiology, Pasteur Institute of Iran, Tehran, Iran \\ 3. Deputy of health, Abadan Faculty of Medical Sciences, Abadan, Iran \\ 4. Department of Epidemiology, School of Public Health, Students Research Center, Hamadan University of Medical Sciences, \\ Hamadan, Iran \\ 5. Associate Professor of Biostatistics, Department of Biostatistics, Hamadan University of Medical Sciences, Hamadan, Iran
}

\section{Artiels Info}

Recieved: 24 May 2016

Accepted: 1 Jul 2016

\section{Keywords}

Tuberculosis

Sputum

Smear

Treatment outcome

\section{Oorerponding huthor}

Salman Khazaei, Department of Epidemiology, School of Public Health, Students Research Center, Hamadan University of Medical Sciences, Hamadan, Iran

Tel: +98936361089

Email: S khazaeii@Yahoo.com

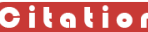

Khazaei S, Yari M, Molaeipour L, Jafari M, Valipour A, Roushanaei G. [Assessing relation between sputum smear grading in positive pulmonary tuberculosis patients with treatment outcome]. Pajouhan Scientific Journal. 2016;14(4):1-7

\section{A b stica $\mathrm{e}$}

Introduction: Unsuccessful treatment of tuberculosis (TB) is big challenge to public health. Some conflicting results in regards with relationship of the sputum smear degree in unsuccessful treatments or death of patients have been reported. Therefore, the current study aimed to evaluate the relationship of the grading of sputum smear-positive patients in the beginning of treatment on treatment consequences.

Methods: In this descriptive-analytic study, which was from cross studies, the records of all positive pulmonary tuberculosis patients were investigated during the years 2010 to 2013 in the Abadan TB register system. Distribution status of sputum positive in the beginning of the treatment was determined by using descriptive statistics. The Chi-square test was used to assess the relationship between sputum smear grading of the patients and treatment outcomes. Data were analyzed using Stata-12.

Results: From a total of 188 patients during the 4 -year period, $68.1 \%$ of them were males and $87.8 \%$ of them were living in urban areas. Furthermore, $80.8 \%$ of the patients recovered, 9.6\% died, 3.2\% had unsuccessful treatment, $3.7 \%$ were transformed to other universities and $2.7 \%$ of them were missing. Also, it was found that there was a significant relationship between the basil level in the beginning of the treatment with treatment outcomes of the patients $(\mathrm{P}=0.02)$. Conclusion: It was found that there was a significance relation between the grading of sputum smear-positive patients and treatment outcomes, as the patients with higher grading of sputum smear-positive the rate of recovery was better. Therefore, it is recommended that appropriate standard treatment under proper conditions and management including daily direct supervision (DOTS) and support of patients by the complete recovery should be offered.

(C) 2016 Pajouhan Scientific Journal. All right reserved 